CERN - AB DEPARTMENT

CERN-AB-2004-095

\title{
MULTITURN EXTRACTION BASED ON TRAPPING IN STABLE ISLANDS
}

\author{
R. Cappi, S. Gilardoni, M. Giovannozzi, M. Martini, E. Métral, A.-S. Müller ${ }^{1)}$, \\ P. Scaramuzzi, R. Steerenberg
}

\begin{abstract}
For some applications an intermediate extraction mode between fast (one turn) and slow (several thousand turns) is needed. This is the case of the five-turn extraction used to transfer the proton beam from the CERN Proton Synchrotron (PS) to the Super Proton Synchrotron (SPS). Unavoidable losses and poor betatronic matching with the receiving machine affect the present approach, which is based on beam slicing by means of an electrostatic septum. These features are rather serious obstacles to an intensity upgrade of the PS/SPS Complex. To overcome these difficulties, a novel extraction technique was proposed recently. By using nonlinear magnetic elements, stable islands can be generated in the transverse phase space. Furthermore, provided the linear tune is varied slowly, it is possible to trap the charged particles inside the stable islands in order to split the beam into different beamlets. Once generated, the distance between these beamlets can be tailored to the extraction needs by simply increasing the distance of the tune from the resonance value. The results of numerical simulations for the novel technique are presented and discussed in detail together with the outcome of the intense experimental studies performed to assess the validity of this approach.
\end{abstract}

Invited paper at the 33rd ICFA Advanced Beam Dynamics Workshop on High Intensity and High Brightness Hadron Beams, Bensheim, Germany, October 18-22, 2004

Geneva, Switzerland

November 17, 2004

\footnotetext{
1) ISS, Forschungszentrum Karlsruhe, Germany
} 


\title{
Multiturn Extraction Based on Trapping in Stable Islands
}

\author{
R. Cappi*, S. Gilardoni*, M. Giovannozzi*, M. Martini*, E. Métral*, P. Scaramuzzi*, \\ R. Steerenberg* and A.-S. Müller ${ }^{\dagger}$ \\ *CERN, AB Department, CH 1211 Geneva 23, Switzerland \\ ${ }^{\dagger}$ FZK-ISS-ANKA, Karlsruhe, Germany
}

\begin{abstract}
For some applications an intermediate extraction mode between fast (one turn) and slow (several thousand turns) is needed. This is the case of the five-turn extraction used to transfer the proton beam from the CERN Proton Synchrotron (PS) to the Super Proton Synchrotron (SPS). Unavoidable losses and poor betatronic matching with the receiving machine affect the present approach, which is based on beam slicing by means of an electrostatic septum. These features are rather serious obstacles to an intensity upgrade of the PS/SPS Complex. To overcome these difficulties, a novel extraction technique was proposed recently. By using nonlinear magnetic elements, stable islands can be generated in the transverse phase space. Furthermore, provided the linear tune is varied slowly, it is possible to trap the charged particles inside the stable islands in order to split the beam into different beamlets. Once generated, the distance between these beamlets can be tailored to the extraction needs by simply increasing the distance of the tune from the resonance value. The results of numerical simulations for the novel technique are presented and discussed in detail together with the outcome of the intense experimental studies performed to assess the validity of this approach.
\end{abstract}

\section{INTRODUCTION}

Since the approval of the CERN Neutrino to Gran Sasso Project (CNGS) [1] and the subsequent efforts devoted to a feasibility study of an intensity upgrade of the PS/SPS complex [2], the special extraction mode, the so-called Continuous Transfer (CT) [3], was reviewed. Such an extraction scheme is required to minimise the filling time of the SPS at $14 \mathrm{GeV} / c$, while reducing the beam emittance so to overcome the aperture limitations at SPS injection. The CT extraction was developed in the seventies [3] with the aim of extracting the beam from the PS in five consecutive turns using an electrostatic septum to slice the beam in the horizontal plane, the tune being 6.25 (see Fig. 1 for a sketch of the principle). The main drawbacks of this technique are the intrinsic losses on the electrostatic septum and the poor betatron matching of the five slices, which might transfer into injection losses in the SPS [4].

Recently, an alternative method was proposed, where the beam is split in the transverse phase space by means of adiabatic capture inside stable islands of the fourth-order resonance [5]. The method was then generalised by using other stable resonances [6].

On the experimental side, intense efforts were devoted to the demonstration of such a novel technique since the year 2002, when beam splitting was observed using a low-intensity single-bunch beam [7]. The key issue, i.e. whether the method would work for a high-intensity

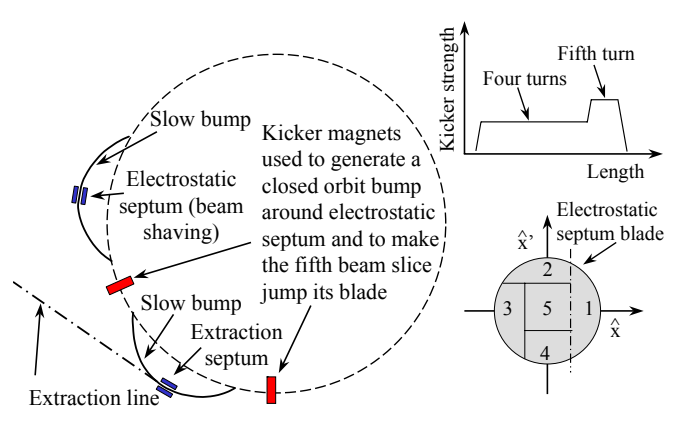

FIGURE 1. Principle of the CT extraction from the PS machine: the extraction scheme (left), the kicker strength as a function of time (upper left), the normalised phase space (lower right).

bunch, was considered during the years 2003 and 2004 (see Refs. $[8,9]$ for more details).

\section{THE NOVEL MULTITURN EXTRACTION}

In the novel approach [5] nonlinear elements such as sextupoles and octupoles are used to generate stable islands in transverse phase space. By varying the horizontal tune, particles can be selectively trapped in the islands by adiabatic capture: some will remain in the phase space area around the origin, while others will migrate to the stable 
islands. As a result, the beam is split into a number of parts in transverse phase space determined by the order of the resonance used, without any mechanical device. The separation between the islands can be controlled so that enough space is available for the beam to jump over a septum blade with almost no particles lost.

A simple model representing the horizontal betatron motion in a circular machine under the influence of sextupole and octupole magnets was used (the motion in the vertical plane can be safely neglected). By assuming that the nonlinear magnets are located at the same place and the single-kick approximation [10] is used, the one-turn transfer map can be expressed as $\mathbf{x}_{n+1}=\mathbf{M}_{n}\left(\mathbf{x}_{n}\right)$ :

$$
\left(\begin{array}{c}
x_{n+1} \\
x^{\prime}{ }_{n+1}
\end{array}\right)=R\left(2 \pi v_{n}\right)\left(\begin{array}{c}
x_{n} \\
x_{n}^{\prime}+x^{2}{ }_{n}+\kappa x^{3}{ }_{n}
\end{array}\right), \kappa=\frac{2}{3} \frac{K_{3}}{\beta_{H} K_{2}^{2}},
$$

where $\left(x, x^{\prime}\right)$ are obtained from the Courant-Snyder coordinates $\left(X, X^{\prime}\right)$ by means of the non-symplectic transformations [10]

$$
\left(x, x^{\prime}\right)=\frac{K_{2} \beta_{H}^{3 / 2}}{2}\left(X, X^{\prime}\right) \quad K_{m}=\frac{L}{B_{0} \rho} \frac{\partial^{m} B_{y}}{\partial x^{m}},
$$

$K_{2,3}$ being the integrated sextupole (octupole) gradient, $L$ the length of the nonlinear element, $B_{y}$ the vertical component of the magnetic field, $B_{0} \rho$ the magnetic rigidity, and $\beta_{H}$ the value of the horizontal beta-function at the location of the nonlinear elements. $R\left(2 \pi v_{n}\right)$ is a $2 \times 2$ rotation matrix of angle $v_{n}$, the fractional part of $Q_{H}$. The map $\mathbf{M}_{n}$ is a time-dependent system through the linear tune. The time-dependence allows varying the phase space topology, thus creating and moving the islands. Also, it allows trapping particles inside the islands. A slow variation of the linear tune, adiabatic with respect to the time scale introduced by the betatron oscillations, pushes particles to cross the separatrix and to be trapped inside the newly-created islands. The trapping process has been simulated by using the model $\mathbf{M}_{n}$ with $\kappa=-1.6$, while the tune $v_{n}$ is varied according to the curve shown in Fig. 2. In the first part, the linear tune is decreased linearly from its initial value of 0.252 to 0.249 . During this part, the capture process takes place. Then, a second linear decrease to the value 0.245 is performed which allows the islands to be moved towards higher amplitudes before extraction.

A set of Gaussian-distributed initial conditions has been generated, and its evolution under the dynamics induced by the map $\mathbf{M}_{n}$ is shown in Fig. 3. The trapping process is clearly visible in the picture: it generates five beamlets, well-separated at the end of the process. No particle is lost during the trapping phase, nor when the islands are moved. Not only the five beamlets have similar surfaces, but also their shape matches the phase space topology

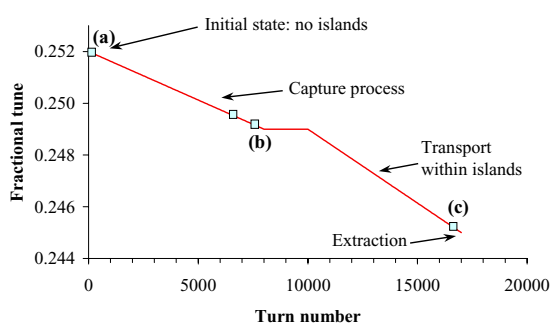

FIGURE 2. Linear tune $v$ as a function of $n$ for the fourthorder resonance.
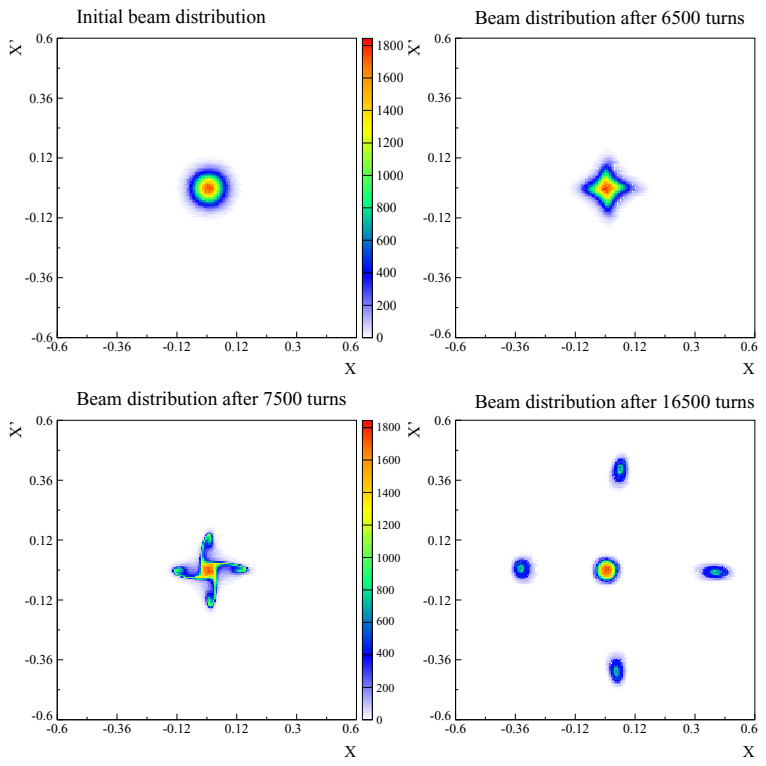

FIGURE 3. Evolution of the beam distribution during the trapping process with four islands. The different plots correspond to tune values represented by solid squares in Fig. 2. Each plot represents $4.9 \times 10^{5}$ points. The initial Gaussian distribution is centred on zero and has $\sigma=0.045$.

very well, making the five parts similar as far as transverse properties are concerned. It is worthwhile pointing out that the first four extracted beamlets have exactly the same emittance, as their shape is dictated by the same phase space structure, i.e. the island along the positive $x$ axis. In this respect, the novel approach proves to be superior to the present CT extraction mode.

\section{MEASUREMENT CAMPAIGN}

\section{Machine and instrumentation}

In the PS machine dedicated sextupoles and octupoles have been installed in sections 21 and 55 (two sextupoles in series in each section) and 20, 21 (two octupoles in series in section 20 and one in section 21) to generate 
the stable islands. Their location is optimised to have at least one island with zero angle at the extraction septum and extraction kicker. Only one set of sextupoles and octupoles can be used at a time. During the measurement campaign in 2002 [7] sextupoles in 21 and octupoles in 20 were used. In 2003, mainly the sextupoles in section 55 and the octupoles in section 20 were powered, while in the year 2004 sextupoles in section 21 and the single octupole in the same section are used. It is worthwhile mentioning that the PS lattice features the minimum of $\beta_{H}$ in even straight sections. A sketch of the PS circumference together with the key elements used in the experiments is shown in Fig. 4.

Phase space reconstruction and beam profile measure-

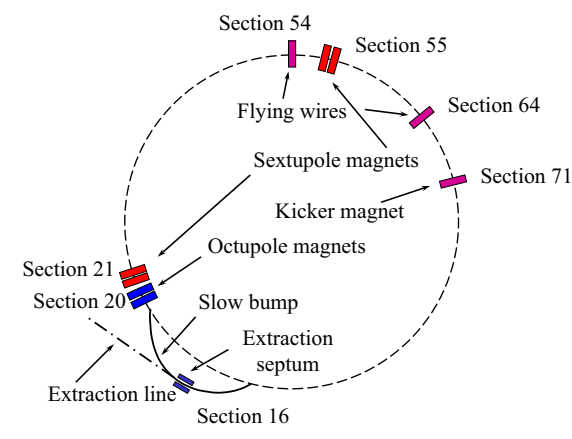

FIGURE 4. Layout of the PS machine including the key elements for the test of adiabatic capture.

ments are the techniques applied in the experimental study. The first ensures that the right phase space topology is generated by the nonlinear magnetic elements, while the latter is meant to record the evolution of the beam distribution during the trapping process and subsequent beam manipulations.

The phase space reconstruction is based on turn-by-turn acquisition of the beam trajectory on two pickups $90^{\circ}$ apart [7]. The beam profile is measured by means of a wire scanner [11]. Among the four installed, two for each transverse plane, the horizontal one in section 54 is routinely used for the measurements reported here. A scintillator is used to detect the secondary particles generated by the beam-wire interaction, thus reconstructing the beam profile.

A summary of beam parameters is reported in Table 1 .

\section{3 measurement campaign}

\section{Phase space measurement}

The low-intensity pencil beam is used for phase space reconstruction to avoid as much as possible beam filamentation. The beam trajectory is perturbed by the kicker magnet normally used for fast beam extraction and betatron oscillations are observed on two pickups $90^{\circ}$ apart.
The pickups selected are those in sections 63 and 67 as

TABLE 1. Main parameters of the beams used for the studies. The value of $\Delta p / p$ refers to $14 \mathrm{GeV} / c$ and $\varepsilon_{H, V}^{*}(\sigma)$ stands for the normalised rms emittance.

\begin{tabular}{|c|c|c|c|c|}
\hline Comments & $\begin{array}{l}\text { Int. } \\
\left(10^{10}\right)\end{array}$ & $\begin{array}{l}\varepsilon_{H}^{*}(\sigma) \\
(\mu \mathrm{m})\end{array}$ & $\begin{array}{l}\varepsilon_{V}^{*}(\sigma) \\
(\mu \mathrm{m})\end{array}$ & $\begin{array}{l}\Delta p / p(\sigma) \\
\left(10^{-3}\right)\end{array}$ \\
\hline pencil beam & 40 & 1.7 & 1.55 & 0.25 \\
\hline low-intensity & 45 & 9 & 2.38 & 0.25 \\
\hline high-intensity* & 600 & 13.2 & 7.6 & 0.6 \\
\hline high-intensity ${ }^{\dagger}$ & 600 & 9.4 & 6.4 & 0.7 \\
\hline
\end{tabular}

* 2003 measurement campaign

2004 measurement campaign

they are in phase with the extraction septum and the extraction kicker.

An example of phase space measurement with a clear signature of stable islands is shown in Fig. 5. Beam posi-
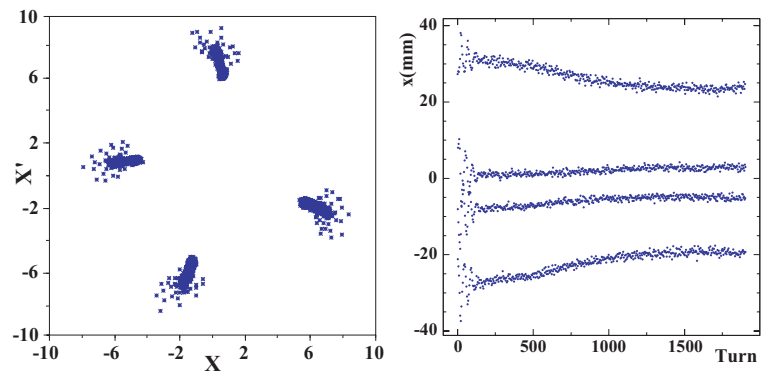

FIGURE 5. Measured horizontal normalised phase space at section 63 (left) and beam position vs. time (right) for the pickup in section 63.

tion oscillations right after the kick indicate that the beam is rotating around the island's centre and filamentation occurs until such oscillations are completely damped. The slow variation over time of the islands' position is very likely due to particles' diffusion outside the islands induced by longitudinal motion. The first few turns can be used to compute the so-called secondary frequency $\omega_{\text {sec }}$ [10], i.e. the betatron frequency around the fixed point. An analytical estimate can be obtained by computing the zero-amplitude frequency for the pendulumlike hamiltonian [12]. The strong component originating from the fourth-order resonance is removed from the measured frequency spectrum by applying a stroboscopic analysis. Then, a refined algorithm to compute the tune [13] is applied. The large set of available data series has been used to derive an average value of the secondary frequency, thus yielding $\omega_{\mathrm{sec}}^{\text {meas }}=(0.0375 \pm$ $0.0008)$, which is in excellent agreement with the theoretical value $\omega_{\mathrm{sec}}^{\mathrm{th}}=0.039$. A refined analysis was also attempted by taking into account the information concerning the different oscillation amplitude inside the island of the various measurement sets. This allowed measuring a detuning curve inside the islands. These data have been compared with numerical simulations and the out- 
come is plotted in Fig. 6. The good agreement is clearly visible, in spite of the measurement errors (probably underestimated). The straight lines represent the linear fit to the data. In the case of the experimental points, a fit weighted with the estimated errors has been applied, yielding $\omega_{\mathrm{sec}}^{\text {meas }}=(0.0408 \pm 0.0009)$, which is even closer to the theoretical result.

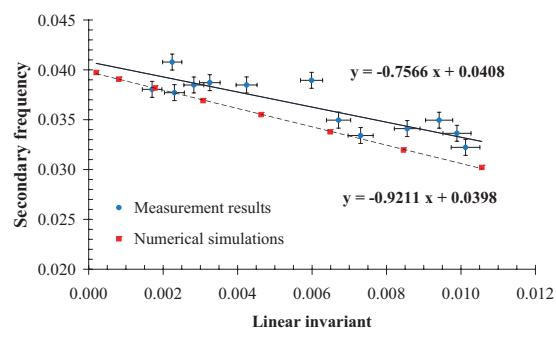

FIGURE 6. Secondary frequency $\omega_{\text {sec }}$ vs. the surface in the normal form space, covered by the orbit inside the island for both experimental and simulation data.

\section{Adiabatic capture of low-intensity bunch}

The first step in the proof of principle of the novel multiturn extraction is the adiabatic capture of a lowintensity beam. To this aim, a beam with a large horizontal emittance, so to simulate the high-intensity beam, and a small vertical emittance to avoid nonlinear coupling between the two transverse planes, is used. No particle loss occurs during the capture and transport process [7].

A series of measurements was performed to assess the actual reversibility of the process by crossing the resonance twice, so to split the beam and merge it back. The resonance crossing time was used as free parameter [9]. Horizontal beam profiles were taken before and after the manipulation to determine whether the initial shape was restored. In Fig. 7 (left) typical beam profiles are shown. The final stage is represented by the superposition of two distribution functions, the second one much larger than the central peak and with tails heavier than a standard Gaussian. More detailed analysis of these data can be found in Ref. [9].

\section{Adiabatic capture of high-intensity bunch}

The most difficult part was the capture of a highintensity bunch of similar characteristics as those required for the proposed intensity upgrade for the CNGS [2]. Indeed, adiabatic trapping conditions were successfully established even for a bunch of intensity up to $625 \times 10^{10}$ protons (the nominal intensity being $600 \times 10^{10}$ protons). An example of the beam profile at the end of the capture process is shown in Fig. 7 (right),
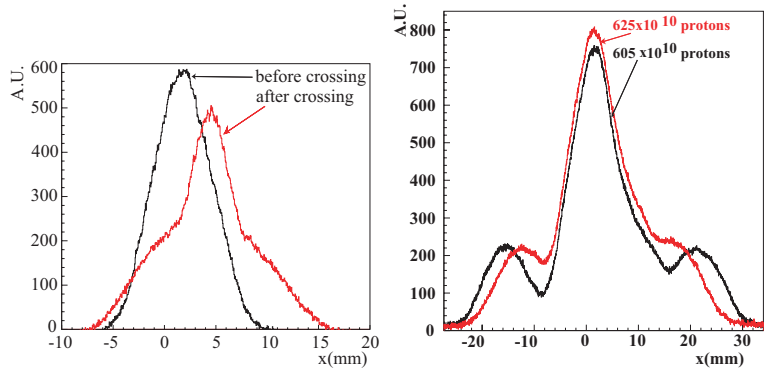

FIGURE 7. Left: Typical beam profiles for reversibility tests with low-intensity beam. Right: Horizontal beam profiles for nominal (black) and record intensity (red). For the latter case, the beamlets separation was reduced to decrease beam losses.

where both cases, i.e. nominal and record intensity, are plotted. Contrary to the low-intensity case, about $20 \%$ of the total beam is lost at the end of the capture process. It was conjectured that this effect might be induced by the strong vertical perturbation generated by the octupoles located at a high- $\beta_{V}$ section, which is imposed by mechanical constraints.

\section{4 measurement campaign}

Based on the experience gained in previous measurement campaigns, an additional octupole was installed during the 2003/2004 shutdown and it was used together with the two sextupoles located in the same section. The activities of phase space reconstruction and capture with the low-intensity beam were successfully resumed, thanks to a very good reproducibility of the PS machine over the years. This also indicates that the proposed approach features a remarkable robustness. The beneficial effect of the new octupole location in a low- $\beta_{V}$ section was reflected in a strong reduction of the losses in the case of capture of the high-intensity bunch: the optimisation of the working point and a proper setting-up of the longitudinal parameters reduced the losses down to $5-10 \%$. However, the real improvement, leading to losses below the sensitivity of the beam transformer, was the dynamical change of the octupole during the capture process as seen in Fig. 8, where the evolution of the current of the key elements is shown. Indeed, by changing the octupole the beamlets can be separated while reducing the interval of tune variation. In Fig. 9 the evolution of the horizontal beam profile at the end of the capture process is reported. The fraction of particles in the beamlets is typically about $13-14 \%$.

Single-turn extraction tests were also performed to assess whether the beamlets could be transported in a transfer line. To reduce losses at the extraction septum the beamlets' separation was reduced and the beam spot 


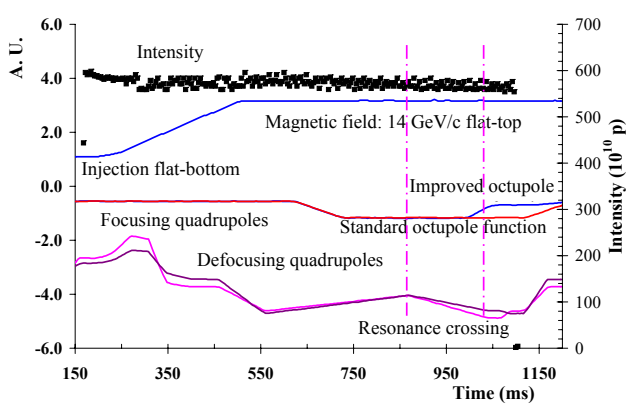

FIGURE 8. Time variation of the main elements used for the adiabatic capture of the high-intensity beam. The beam intensity is clearly constant throughout the whole splitting process.
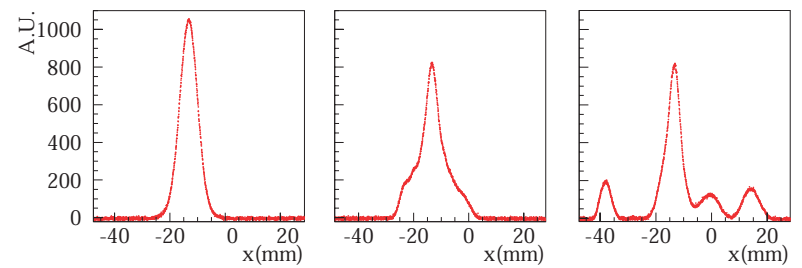

FIGURE 9. Horizontal beam profile measured by the wire scanner for three values of the tune.

recorded in the transfer line via an Optical Transition Radiation (OTR) monitor (see Fig. 10). In the $(x, y)$ space

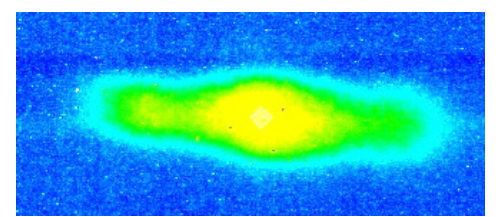

FIGURE 10. Two-dimensional view of the beam in the physical space $(x, y)$ in the transfer line downstream of the PS machine.

two beamlets are projected onto the beam core, while the two lateral beamlets are slightly separated, thus originating the structure with three spots.

\section{CONCLUSIONS}

A novel approach to perform multiturn extraction was proposed a few years ago. Indeed, it is worthwhile stressing that such a technique allows manipulating the transverse emittance in a synchrotron, thus broadening the spectrum of possible beam manipulations.

Following the successful results of numerical simulations, an experimental campaign was launched in the year 2002. Presently, a single-bunch proton beam can be split in the horizontal plane with no measurable losses both at low- and high-intensity. The generated beamlets can be sufficiently separated to allow a virtually loss-free extraction. A number of issues are still under discussion before the proposed method can be considered a viable solution to the present Continuous Transfer. In particular, the fraction of trapped particles should be increased from the achieved value of $13-14 \%$ to the nominal value of $20 \%$. Moreover, extraction tests proper are probably not possible with the present hardware and should be postponed to a later stage.

Finally, it is worth mentioning that studies aimed at applying this approach to multiturn injection are in progress and they seem to suggest that it should be possible generating hollow bunches in the transverse phase plane.

\section{ACKNOWLEDGEMENTS}

We would like to thank M. E. Angoletta for support with the turn-by-turn acquisition system, M. Benedikt and $\mathrm{M}$. Chanel for preparing the various beams in the PS-Booster, S. Hancock for setting up the longitudinal parameters in the PS and C. Bal, B. Dehning, J. Koopman, U. Raich for the support with the wire scanner.

\section{REFERENCES}

1. Elsener, K., (Ed.) et al., CERN 98-02 (1998).

2. Cappi, R., (Ed.) et al., CERN-PS (AE) 2001-041 (2001).

3. Bovet, C., Fiander, D., Henny, L., Krusche, A., Plass, G., in 1973 Particle Accelerator Conference, edited by Dupen,D. W., (IEEE, New York), 1973, p. 438.

4. Cappi, R., and Giovannozzi, M., CERN-PS (AE) 2002-083 (2002)

5. Cappi, R., Giovannozzi, M., Phys. Rev. Lett. 88, 104801, (2002).

6. Cappi, R., Giovannozzi, M., Phys. Rev. ST Accel. Beams 7, 024001, (2002).

7. Cappi, R., Giovannozzi, M., Martini, M., Métral, E., Müller, A.-S., and Steerenberg, R., in PAC2003 Conference, edited by Chew, J., Lucas, P., and Webber, S. (IEEE Computer Society Press, Piscataway), 2003, p. 388.

8. Giovannozzi, M., (Ed.) et al., CERN-AB-2004-003-ABP (2004).

9. Giovannozzi, M., Cappi, R., Gilardoni, S., Martini, M., Métral, E., Sakumi, A., Steerenberg, R., Müller, A.-S., in Ninth European Particle Accelerator Conference, edited by Poole, J., and Petit-Jean-Genaz, C., (Institute of Physics, London UK), 2004, p. 175.

10. Bazzani, A., Todesco, E., Turchetti, G., and Servizi, G., CERN 1994-02 (1994).

11. Steinbach, C., Van Rooij, M., IEEE Trans. Nucl. Sci. 32, (1985) p. 1920.

12. Giovannozzi, M., Scaramuzzi, P., in Ninth European Particle Accelerator Conference, edited by Poole, J., and Petit-Jean-Genaz, C., (Institute of Physics, London UK), 2004, p. 1858.

13. Bartolini, R., Bazzani, A., Giovannozzi, M., Scandale, W., and Todesco, W., Part. Accel. 52, 147, (1995). 